\title{
Bayes and Invariante Estimation of Parameters Under a Bounded Asymmetric Loss Function
}

\author{
N. Sanjari Farsipour \\ Department of Statistics, Faculty of Mathematical sciences, Alzahra University, Tehran, Iran \\ Email: sanjari_n@yahoo.com
}

\begin{abstract}
In this paper we consider the estimation of parameters under a bounded asymmetric loss function. The Bayes and invariant estimator of location and scale parameters in the presence and absence of a nuisance parameter is considered. Some examples in this regard are included.
\end{abstract}

Keywords: Bayes estimation; invariance; location parameter; scale parameter; bounded Asymmetric loss.

\section{$1 \quad$ Introduction}

In the literature, the estimation of a parameter is usually considered when the loss is squared error or in general any convex and symmetric function. The quadratic loss function has been criticized by some researches (e.g., [4], [5], [6] and [7]). The proposed loss function is

$$
L(\delta, \theta)=k\left\{1-e^{b\left\{1+a(\delta-\theta)-e^{a(\delta-\theta)}\right\}}\right\}
$$

where $a \neq 0$ determines the shape of the loss function, $b>0$ serves to scale the loss and $k>0$ is the maximum loss parameter. The general form of the loss function is illustrated in Figure 1. This is obviously a bounded asymmetric loss function.

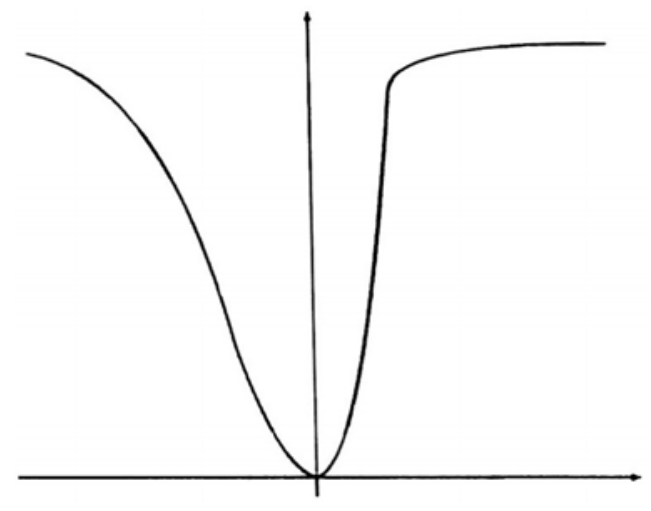

Figure 1. The loss function (1.1) for $a=1$.

In this paper, we first study the problem of estimation of a location parameter, using the loss function (1.1). In section 2 we introduce the best location-invariant estimator of $\theta$ under the loss (1.1). In section 3, Bayesian estimation of the normal mean is obtained under the loss (1.1). Then we study the problem of estimation of a scale parameter, using the loss function

$$
L(\delta, \tau)=k\left\{1-e^{b\left\{1+a\left(\frac{\delta}{\tau}-1\right)-e^{a\left(\frac{\delta}{\tau}-1\right)}\right\}}\right\}
$$

where $a \neq 0, b, k>0$. The loss (1.2) is scale invariant and bounded. In section 4 we introduce the best invariant estimator of the scale parameter $\tau$ under the loss (1.2). Finally in section 5 we consider a 
subclass of the exponential family and obtain the Bayes estimates of $\tau$ under the loss (1.2). Since the parameters $b$ and $k$ do not have any influence on our results, so without loss of generality we take $b=k=1$ in the rest of the paper.

\section{Best Location-Invariant Estimator}

Let $\mathbf{X}=\left(X_{1}, \ldots, X_{n}\right)$ have a joint distribution with probability density $f(\mathbf{X}-\theta)=f\left(X_{1}-\theta, \ldots, X_{n}-\theta\right)$ where $f$ is known and $\theta$ is an unknown location parameter. The class of all location invariant estimators of a location parameter $\theta$ is of the form [3]

$$
\delta(\mathbf{X})=\delta_{0}(\mathbf{X})-v(\mathbf{Y})
$$

where $\delta_{0}$ is any location-invariant estimator and $\mathbf{Y}=\left(Y_{1}, \ldots, Y_{n-1}\right)$ with $Y_{i}=X_{i}-X_{n}, \quad i=1, \ldots, n-1$ and the best location-invariant estimator $\delta^{*}$ of $\theta$ under the loss function(1.1), is $\delta^{*}(\mathbf{X})=\delta_{0}(\mathbf{X})-v^{*}(\mathbf{y})$, where $v^{*}(\mathbf{y})$ is a number which minimizes

$$
E_{\theta=0}\left[1-e^{1+a\left(\delta_{0}(\mathbf{X})-v(\mathbf{y})\right)-e^{a\left(\delta_{0}(\mathbf{X})-v(\mathbf{y})\right)}} \mid \mathbf{Y}=\mathbf{y}\right]
$$

(see [3]). Differentiating with respect to $v(\mathbf{y})$ and equating to zero, it can be seen that $v^{*}(\mathbf{y})$ must satisfy the following equation

$$
E_{\theta=0}\left[\left(e^{a\left(\delta_{0}(\mathbf{X})-v^{*}(\mathbf{y})\right)}-1\right) e^{a\left(\delta_{0}(\mathbf{X})-v^{*}(\mathbf{y})\right)-e^{a\left(\delta_{0}(\mathbf{X})-v^{*}(\mathbf{y})\right)}} \mid \mathbf{Y}=\mathbf{y}\right]=0
$$

Example 2.1: (normal mean) Let $X_{1}, \ldots, X_{n}$ be i.i.d. random variables having normal distribution with mean $\theta$ (real but unknown) and known variance $\sigma^{2}$. If $\delta_{0}(\mathbf{X})=\bar{X}$, it follows from Basu's theorem that $\delta_{0}(\mathbf{X})$ is independent of $\mathbf{Y}$ and hence the best location-invariant estimator of $\theta$ is given by $\delta^{*}(\mathbf{X})=\bar{X}-v^{*}$, when $v^{*}$ is a number which satisfies (2.1), i.e.

$$
\int_{-\infty}^{\infty} e^{-\frac{n}{2 \sigma^{2}}\left(\mathbf{x}-\frac{2 a \sigma^{2}}{n}\right)^{2}-e^{a \mathbf{x}-a v^{*}}} \mathrm{~d} x=e^{a v^{*}-\frac{a^{2} \sigma^{2}}{n}} \int_{-\infty}^{\infty} e^{-\frac{n}{2 \sigma^{2}}\left(\mathbf{x}-\frac{a \sigma^{2}}{n}\right)^{2}-e^{a \mathbf{x}-a v^{*}}} \mathrm{~d} x
$$

So, we can find $v^{*}$ by a numerical solution.

Example 2.2: (Uniform) Let $X_{1}, \ldots, X_{n}$ be i.i.d. according to the uniform distribution on $\left(\theta-\frac{\beta}{2}, \theta+\frac{\beta}{2}\right)$ where $\theta$ is real (but unknown) and $\beta(>0)$ is known. Taking $\delta_{0}(\mathbf{X})=\left(X_{(1)}+X_{(2)}\right) / 2$ which is an invariant estimator of $\theta$, the conditional distribution of $\delta_{0}(\mathbf{X})$ given $\mathbf{Y}=\mathbf{y}$ depends on $\mathbf{y}$ only through differences $X_{(i)}-X_{(1)}=V_{i}, i=2, \ldots, n$. Now, note that $\left(X_{(1)}, X_{(\mathrm{n})}\right)$ is a complete sufficient statistic for $(\theta, \beta)$ and is independent of $Z_{i}=\frac{X_{(i)}-X_{(1)}}{X_{(\mathrm{n})}-X_{(1)}}, i=2, \ldots, n-1$ for all $\theta, \beta$ by Basu's theorem. Hence $\left(X_{(1)}, X_{(n)}\right)$ and $Z_{i}^{\prime} s$ are independent for all $\theta$ and any given $\beta$. Also, note that the conditional distribution of $\delta_{0}(\mathbf{X})$ given $V_{i}^{\prime} s$ which is equivalent to conditional distribution of $\delta_{0}(\mathbf{X})$ given $X_{(\mathrm{n})}-X_{(1)}$ and $Z_{i}^{\prime} s$ depends only on $X_{(\mathrm{n})}-X_{(1)}$. On the other hand, the conditional distribution of $\delta_{0}(\mathbf{X})$ given $W=X_{(n)}-X_{(1)}$ at $\theta=0$ is of the form

$$
f_{\delta_{0}(\mathbf{X}) \mid W=w}(t)=\frac{1}{\beta-w} \quad \text { if }|t|<\frac{\beta-w}{2} ; \beta>w
$$


Hence the estimator $\delta^{*}(\mathbf{X})=\frac{X_{(1)}+X_{(n)}}{2}-v^{*}$ is the MRE estimator of $\theta$, if $v^{*}$ satisfies (2.1), which simplifies to

$$
e^{-a\left(\frac{\beta-w}{2}+v^{*}\right)-e^{-a\left(\frac{\beta-w}{2}+v^{*}\right)}}-e^{a\left(\frac{\beta-w}{2}-v^{*}\right)-e^{a\left(\frac{\beta-w}{2}-v^{*}\right)}}=(1+a) e^{-e^{a\left(\frac{\beta-w}{2}-v^{*}\right)}}-(1+a) e^{-e^{-a\left(\frac{\beta-w}{2}+v^{*}\right)}}
$$

So, we can find $v^{*}$ by a numerical solution.

Example 2.3: (Exponential distribution) Let $X_{1}, \ldots, X_{n}$ be i.i.d. random variables with the density

$$
f_{\theta}(x)=\frac{1}{\beta} e^{-(x-\theta) / \beta} \quad x \geq \theta
$$

where $\theta \in R$ is unknown and $\beta(>0)$ is known. $\delta_{0}(\mathbf{X})=X_{(1)}$ is an equivariant estimator and by the Basu's theorem, it is independent of $\mathbf{Y}$. Therefore, $\delta^{*}(\mathbf{X})=X_{(1)}-v^{*}$ is the MRE estimator of $\theta$, if $v^{*}$ satisfies (2.1), i.e. satisfies

$$
\begin{aligned}
& \int_{0}^{e^{-a v^{*}}} x^{1-\frac{n}{a \beta}} e^{-x} \mathrm{~d} x=e^{\frac{n v^{*}}{\beta}} \int_{0}^{e^{-a v^{*}}} x^{-\frac{n}{a \beta}} e^{-x} \mathrm{~d} x \quad ; a<0 \\
& \int_{e^{-a{ }^{*}}}^{\infty} x^{1-\frac{n}{a \beta}} e^{-x} \mathrm{~d} x=e^{\frac{n \nu^{*}}{\beta}} \int_{e^{-a v^{*}}}^{\infty} x^{-\frac{n}{a \beta}} e^{-x} \mathrm{~d} x \quad ; a>0
\end{aligned}
$$

which simplifies to

$$
\sum_{r=0}^{1-\frac{n}{a \beta}} \frac{\left(1-\frac{n}{a \beta}\right) !}{\left(1-\frac{n}{a \beta}-r\right) !} e^{a v^{*}\left(1-\frac{n}{a \beta}-r\right)}=e^{\frac{a v^{*}}{\beta}} \sum_{r=0}^{-\frac{n}{a \beta}} \frac{\left(-\frac{n}{a \beta}\right) !}{\left(-\frac{n}{a \beta}-r\right) !} e^{a v^{*}\left(\frac{n}{a \beta}+r\right)}
$$

So, we can find $v^{*}$ by a numerical solution.

\section{Bayes Estimation of the Normal Mean}

Let $X_{1}, \ldots, X_{n}$ be a random sample of size $n$ from a normal distribution with mean $\theta$ (unknown parameter) and variance $\sigma^{2}$ (known parameter). In this section we consider Bayesian estimation of the parameter $\theta$ using the loss function (1.1).

If the conjugate family of prior distributions for $\theta$ is the family normal distributions $N\left(\mu, b^{2}\right)$, then the posterior distribution of $\theta$ is $N(m, v)$ where

$$
m=\frac{\frac{n \bar{x}}{\sigma^{2}}+\frac{\mu}{b^{2}}}{\frac{n}{\sigma^{2}}+\frac{1}{b^{2}}} \quad \& \quad v=\frac{1}{\frac{n}{\sigma^{2}}+\frac{1}{b^{2}}},
$$

and the posterior risk of an estimator $\delta(\mathbf{X})$ under the loss function (1.1) is

$$
\left\{1-E\left[e^{1+a(\theta-\delta(\mathbf{X}))-e^{a(\theta-\delta(\mathbf{x}))}} \mid \mathbf{X}\right]\right\}=1-\int_{-\infty}^{\infty} e^{1+a(\theta-\delta(\mathbf{X}))-e^{a(\theta-\delta(\mathrm{x}))}} \frac{1}{\sqrt{n \pi \nu}} e^{-\frac{1}{2 v}(\theta-m)^{2}} \mathrm{~d} \theta
$$

so, $\delta_{B}(\mathbf{X})$ is the solution of the following integral equation

$$
\int_{-\infty}^{\infty} e^{-\frac{1}{2 v}(\theta-2 a v-m)^{2}-e^{a\left(\theta-\delta_{B}\right)}} \mathrm{d} \theta=e^{a\left(\delta_{B}-a v-m\right)} \int_{-\infty}^{\infty} e^{-\frac{1}{2 v}(\theta-a v-m)^{2}-e^{a\left(\theta-\delta_{B}\right)}} \mathrm{d} \theta
$$

Hence, we can find $\delta_{B}$ from the equation (3.1) by a numerical solution. 
Also, notice that the generalized Bayes estimator relative to a diffuse prior, $\pi(\theta)=1$ for all $\theta \in R$ can be found by letting $b \rightarrow \infty$, i.e. $v \rightarrow \frac{\sigma^{2}}{n}$.

In the presence of a nuisance parameter $\sigma^{2}$, i.e. when $\sigma^{2}$ is unknown, a modified loss function is as follows

$$
L(\delta ; \theta, \sigma)=1-e^{1+a\left(\frac{\delta-\theta}{\sigma}\right)-e^{a\left(\frac{\delta-\theta}{\sigma}\right)}}
$$

$a \neq 0$ which is a location scale invariant loss function.

In this position, we obtain a class of Bayes estimators of the location parameter $\theta$. Let $\tau=\frac{1}{\sigma^{2}}$ be the precision which is unknown and suppose that conditional on $\tau, \theta$ has a normal distribution with mean $\mu$ and variance $1 / \lambda \tau$, where $\mu \in R, \lambda>0$ are both known constants, i.e., $\theta \mid \tau \sim N\left(\mu, \frac{1}{\lambda \tau}\right)$ and $\tau$ has a p.d.f $\mathrm{g}(\tau)$. In this case, one can easily verify that

$$
\pi(\theta \mid \mathbf{x}, \tau) \propto e^{-\frac{r}{2} \sum_{i=1}^{n}\left(x_{i}-\theta\right)^{2}} e^{-\frac{r \lambda}{2}(\theta-\mu)^{2}}
$$

Or

$$
\pi(\theta \mid \mathbf{x}, \tau) \propto \exp \left\{-\frac{\tau}{2}(n+\lambda)\left[\theta-\left(\frac{n}{n+\lambda} \bar{x}+\frac{\lambda}{n+\lambda} \mu\right)\right]^{2}\right\}
$$

It is clear that $\theta \mid \mathbf{x}, \tau \sim N\left(\eta, \frac{1}{\tau(n+\lambda)}\right)$, with $\eta=\frac{n}{n+\lambda} \bar{x}+\frac{\lambda}{n+\lambda} \mu$. To obtain the Bayes estimate of $\theta$ for our problem, it is enough to find an estimate $\delta(x)$ which minimizes $E[L(\delta(\mathbf{X}) ; \theta, \tau) \mid \mathrm{X}, \tau]$ for any $\mathrm{X}, \tau$. This expectation is under the distribution of $\theta \mid \mathrm{X}, \tau$. So $\delta_{B}$ is the solution of the following integral equation

$\int_{0}^{\infty} \int_{-\infty}^{\infty} e^{a \sqrt{\tau}\left(\theta-\delta_{B}\right)-e^{a \sqrt{\tau}\left(\theta-\delta_{B}\right)-\frac{r}{2}(n+\lambda)(\theta-\eta)^{2}}} g(\tau) \mathrm{d} \theta \mathrm{d} \tau=\int_{0}^{\infty} \int_{-\infty}^{\infty} e^{2 a \sqrt{\tau}\left(\theta-\delta_{B}\right)-e^{a \sqrt{\tau}\left(\theta-\delta_{B}\right)-\frac{r}{2}(n+\lambda)(\theta-\eta)^{2}}} g(\tau) \mathrm{d} \theta \mathrm{d} \tau$

which can be solved numerically.

\section{Best Scale Invariant Estimator}

Consider a random sample $X_{1}, \ldots, X_{n}$ from $\frac{1}{\tau} f\left(\frac{\mathbf{x}}{\tau}\right)$, where $f$ is a known function, and $\tau$ is an unknown scale parameter. It is desired to estimate $\tau$ under the loss function (1.2). The class of all scale-invariant estimators of $\tau$ is of the form

$$
\delta(\mathbf{X})=\delta_{0}(\mathbf{X}) / W(\mathbf{Z})
$$

where $\delta_{0}$ is any scale-invariant estimator, $\mathbf{X}=\left(X_{1}, \ldots, X_{n}\right)$, and $\mathbf{Z}=\left(Z_{1}, \ldots, Z_{n}\right) \quad$ with $\quad Z_{i}=\frac{X_{i}}{X_{n}}$; $i=1, \ldots, n-1, Z_{n}=\frac{X_{n}}{\left|X_{n}\right|}$. Moreover the best scale-invariant (minimum risk equivariant (MRE)) estimator $\delta^{*}$ of $\tau$ is given by

$$
\delta^{*}(\mathbf{X})=\delta_{0}(\mathbf{X}) / w^{*}(\mathbf{Z})
$$

where $w^{*}(\mathbf{Z})$ is a function of $\mathbf{Z}$ which maximizes 


$$
E_{\tau=1}\left[e^{1+a\left(\frac{\delta_{0}(\mathrm{X})}{\mathrm{w}(\mathrm{Z})}-1\right)-e^{a\left(\frac{\delta_{0}(\mathrm{X})}{\mathrm{w}(\mathrm{Z})}-1\right)}} \mid \mathbf{Z}=\mathbf{z}\right]
$$

In the presence of a location parameter as a nuisance parameter, the MRE estimator of $\tau$ is of the form

$$
\delta^{*}(\mathbf{X})=\delta_{0}(\mathbf{Y}) / w^{*}(\mathbf{Z})
$$

where $\delta_{0}(\mathbf{Y})$ is any finite risk scale-invariant estimator of $\tau$, based on $\mathbf{Y}=\left(\mathrm{Y}_{1}, \ldots, \mathrm{Y}_{n-1}\right)$, with $Y_{i}=X_{i}-X_{n} ; i=1, \ldots, n-1, \quad \mathbf{Z}=\left(Z_{1}, \ldots, Z_{n-1}\right), Z_{i}=\frac{Y_{i}}{Y_{n-1}} ; i=1, \ldots, n-2, \quad$ and $\quad Z_{n-1}=\frac{Y_{n-1}}{\left|Y_{n-1}\right|}$ and $w^{*}(\mathbf{Z})$ is any function of $\mathbf{Z}$ maximizing

$$
E_{\tau=1}\left[e^{1+a\left(\frac{\delta_{0}(\mathrm{Y})}{\mathrm{w}(\mathrm{Z})}-1\right)-e^{a\left(\frac{\delta_{0}(\mathrm{Y})}{\mathrm{w}(\mathrm{Z})}-1\right)}} \mid \mathbf{Z}=\mathbf{Z}\right]
$$

In many cases, when $\tau=1$, we can find an equivariant estimator $\delta_{0}(\mathbf{X})$ or $\delta_{0}(\mathbf{Y})$ which has the gamma distribution with known parameters $v, \eta$ and is independent of $\mathbf{Z}$.

It follows that $\delta^{*}=\frac{\delta_{0}}{w^{*}}$ is the MRE estimator of $\tau$ where $w^{*}$ is a number which maximizes

$$
g(w)=\int_{0}^{\infty} e^{1+a\left(\frac{x}{w}-1\right)-e^{a\left(\frac{x}{w}-1\right)}} \frac{\eta^{v} x^{\nu-1}}{\Gamma(v)} e^{-\eta x} \mathrm{~d} x=\frac{\eta^{\nu}}{\Gamma(\boldsymbol{v})} e^{1-a} \int_{0}^{\infty} x^{\nu-1} e^{x\left(\frac{a}{w}-\eta\right)-e^{a\left(\frac{x}{w}-1\right)}} \mathrm{d} x
$$

and hence $w^{*}$ must satisfy the following equation

$$
\int_{0}^{\infty} x^{\nu-1} e^{\left(\frac{2 a}{w^{*}}-\eta\right) x-e^{\frac{a x}{w^{*}}-a}} \mathrm{~d} x=e^{a} \int_{0}^{\infty} x^{v} e^{\left(\frac{a}{w^{*}}-\eta\right) x-e^{\frac{a x}{w^{*}}-a}} \mathrm{~d} x
$$

Theorem 4.1: If $\delta_{0}(\mathrm{X})$ is a finite risk scale-invariant estimator of $\tau$, which has the gamma distribution with known parameters $v, \eta$, when $\tau=1$. Then the MRE (minimum risk equivariant) estimator of $\tau$ under the loss function (1.2) is $\delta^{*}(\mathbf{X})=\frac{\delta_{0}(\mathbf{X})}{w^{*}}$, where $w^{*}$ must satisfy the equation (4.4).

Example 4.1: (Exponential) Let $X_{1}, \ldots, X_{n}$ be a random sample from $E(0, \lambda)$ with density $\frac{1}{\lambda} e^{-\frac{x}{\lambda}} ; x>0$, and consider the estimation of $\lambda$ under the loss (1.2). $\delta_{0}(\mathbf{X})=\sum_{i=1}^{n} X_{i}$ is an equivariant estimator which has $\mathrm{Ga}(\mathrm{n}, 1)$-distribution when $\lambda=1$ and it follows from the Basu's theorem that $\delta_{0}$ is independent of $\mathbf{Z}$, hence the MRE estimator of $\lambda$ under the loss $(1.2)$ is $\delta^{*}(\mathbf{X})=\frac{\sum_{i=1}^{n} X_{i}}{\omega^{*}}$, where $\omega^{*}$ must satisfy the following equation

$$
\int_{0}^{\infty} x^{n-1} e^{\left(\frac{2 a}{\left.w^{*}-1\right) x-e^{\frac{a x}{w^{*}}-a}}\right.} \mathrm{d} x=e^{a} \int_{0}^{\infty} x^{n} e^{\left(\frac{a}{w^{*}}-1\right) x-e^{\frac{a x}{w^{*}}-a}} \mathrm{~d} x
$$


Example 4.1: (Continued) Suppose that $X_{1}, \ldots, X_{n}$ is a random sample of $E(\theta, \lambda)$ with density $\frac{1}{\lambda} e^{-(x-\theta) / \lambda} ; x>\theta$, and consider the estimation of $\lambda$ when $\theta$ is unknown. We know that $\left(X_{(1)}, \sum_{i=1}^{n}\left(\mathrm{X}_{i}-X_{(1)}\right)\right) \quad$ is a complete sufficient statistics for $(\theta, \lambda)$. It follows that $\delta_{0}(\mathbf{Y})=2 \sum_{i=1}^{n}\left(X_{i}-X_{(1)}\right)$ has $\mathrm{Ga}\left(\mathrm{n}-1, \frac{1}{2}\right)$-distribution, when $\lambda=1$, and from the Basu's theorem $\delta_{0}(\mathbf{Y})$ is independent of $\mathbf{Z}$ and hence $\delta^{*}(\mathbf{X})=\frac{\sum_{i=1}^{n}\left(X_{i}-X_{(1)}\right)}{\omega^{*}}$ is the MRE estimator of $\lambda$ under the loss (1.2), where $\omega^{*}$ must satisfy the following equation

$$
\int_{0}^{\infty} x^{n-2} e^{\left(\frac{2 a}{w}-\frac{1}{2}\right) x-e^{\frac{a x}{w}-a}} \mathrm{~d} x=e^{a} \int_{0}^{\infty} x^{n-1} e^{\left(\frac{a}{w}-\frac{1}{2}\right) x-e^{\frac{a x}{w}-a}} \mathrm{~d} x
$$

Example 4.2: (Normal variance) Let $X_{1}, \ldots, X_{n}$ be a random sample of $N\left(0, \sigma^{2}\right)$ and consider the estimation of $\sigma^{2} \cdot \delta_{0}(\mathbf{X})=\sum_{i=1}^{n} X_{i}^{2}$ is a finite risk scale-invariant estimator of $\sigma^{2}$ and is independent of $\mathbf{Z}$, and when $\sigma^{2}=1, \delta_{0}(\mathbf{X})$ has $\mathrm{Ga}\left(\frac{n}{2}, \frac{1}{2}\right)$-distribution and hence $\delta^{*}(\mathbf{X})=\frac{\sum_{i=1}^{n} X_{i}^{2}}{\omega^{*}}$ is the MRE estimator of $\sigma^{2}$, where $\omega^{*}$ must satisfy the following equation

$$
\int_{0}^{\infty} x^{\frac{n}{2}-1} e^{\left(\frac{2 a}{\left.w^{*}-\frac{1}{2}\right) x-e^{\frac{a x}{w^{*}}-a}}\right.} \mathrm{d} x=e^{a} \int_{0}^{\infty} x^{\frac{n}{2}} e^{\left(\frac{a}{\left.w^{*}-\frac{1}{2}\right)-e^{\frac{a x}{w^{*}}-a}}\right.} \mathrm{d} x
$$

Example 4.2: (Continued) Let $X_{1}, \ldots, X_{n}$ be a random sample from $N\left(\mu, \sigma^{2}\right)$, with a nuisance parameter $\mu$. In estimating $\sigma^{2}$ using the loss (1.2), it follows that $\delta_{0}(\mathbf{X})=\sum_{i=1}^{n}\left(X_{i}-\bar{X}\right)^{2}$ is independent of $\mathbf{Z}$, and when $\sigma^{2}=1$, the distribution of $\delta_{0}(\mathbf{Y})$ is $\operatorname{Ga}\left(\frac{n-1}{2}, \frac{1}{2}\right)$. Therefore, $\delta^{*}(\mathbf{X})=\frac{\sum_{i=1}^{n}\left(X_{i}-\bar{X}\right)^{2}}{\omega^{*}}$ is the MRE estimator of $\sigma^{2}$, where $\omega^{*}$ must satisfy the following equation

$$
\int_{0}^{\infty} x^{\frac{n-3}{2}} e^{\left(\frac{2 a}{\left.w^{*}-\frac{1}{2}\right) x-e^{\frac{a x}{w^{*}}-a}}\right.} \mathrm{d} x=e^{a} \int_{0}^{\infty} x^{\frac{n-1}{2}} e^{\left(\frac{a}{\left.w^{*}-\frac{1}{2}\right) x-e^{\frac{a x}{w^{*}}-a}}\right.} \mathrm{d} x
$$

Example 4.3: (Inverse Gaussian with zero drift) Let $X_{1}, \ldots, X_{n}$ be a random sample of $\operatorname{IG}(\infty, \lambda)$ with density

$$
f(x \mid \lambda)=\left(\frac{\lambda}{2 \pi x^{3}}\right)^{\frac{1}{2}} e^{-\frac{\lambda}{2 x}} \quad \text { if } x>0
$$

and consider the estimation of $\lambda . \delta_{0}(\mathbf{X})=\sum_{i=1}^{n} X_{i}^{-1}$ has $\operatorname{Ga}\left(\frac{n}{2}, \frac{1}{2}\right)$-distribution and is independent of $\mathbf{Z}$ and hence $\delta^{*}(\mathbf{X})=\frac{\sum_{i=1}^{n} X_{i}^{-1}}{\omega^{*}}$ is the MRE estimator of $\lambda$, where $\omega^{*}$ must satisfy the equation (4.7). 


\section{$5 \quad$ Bayes Estimation of Scale Parameters}

In the section, we consider the Bayesian estimation of the scale parameter $\tau$ in a subclass of oneparameter exponential families in which the complete sufficient statistic $\delta_{0}(\mathbf{X})$ has $\mathrm{G}\left(\boldsymbol{v}, \frac{\eta}{2}\right)$ distribution, where $v>0, \eta>0$ are known.

Assume that the conjugate family of prior distributions for $\beta=\frac{1}{\tau}$ is the family of Gamma distribution $\operatorname{Ga}(\alpha, \xi)$. Now, the posterior distribution of $\beta$ is $\mathrm{Ga}\left(v+\alpha, \xi+\eta \delta_{0}(\mathrm{x})\right)$ and the Bayes estimate of $\tau$ is a function $\delta(\mathrm{x})$ which maximizes the function

$$
E\left[e^{1+a(\beta \delta-1)-e^{a(\beta \delta-1)}} \mid \mathbf{X}\right]=\frac{\left(\eta \delta_{0}(\mathbf{X})+\xi\right)^{\nu+\alpha}}{\Gamma(\nu+\alpha)} e^{1-a} \int_{0}^{\infty} \beta^{\nu+\alpha-1} e^{\left(a \delta-\xi-\eta_{0}(\mathbf{X})\right) \beta-e^{a(\beta \delta-1)}} \mathrm{d} \beta
$$

Hence, the maximized $\delta$ must satisfy the following integral equation,

$$
\int_{0}^{\infty} \beta^{\nu+\alpha} e^{\left(2 \mathrm{a} \delta-\xi-\eta \delta_{0}(\mathrm{x})\right) \beta-e^{a(\beta \delta-1)}} \mathrm{d} \beta=e^{a} \int_{0}^{\infty} \beta^{\nu+\alpha} e^{\left(\mathrm{a} \delta-\xi-\eta \delta_{0}(\mathrm{x})\right) \beta-e^{a(\beta \delta-1)}} \mathrm{d} \beta
$$

So all estimators satisfying (5.1) are also Bayes estimators.

Example 5.1: (Fisher Nile's problem) The classical example of an ancillary statistic is known as the problem of Nile, originally formulated by Fisher [1]. Assume that $X$ and $Y$ are two positive valued random variables with the joint density function

$$
f(x, y ; \tau)=e^{-\left(\tau x+\frac{1}{\tau} y\right)} \quad ; x>0, y>0, \tau>0
$$

and that $\left(X_{i}, Y_{i}\right), i=1, \ldots, n$ is a random sample of $n$ paired observation on $(X, Y)$. Let $\bar{X}=\frac{1}{n} \sum_{i=1}^{n} X_{i}$, $\bar{Y}=\frac{1}{n} \sum_{i=1}^{n} Y_{i}, T=\sqrt{\frac{\bar{Y}}{\bar{X}}}, u=\sqrt{\bar{X} \bar{Y}} . T$ is the MLE of $\tau$ and the pair $(T, U)$ is a jointly sufficient, but not complete statistics for $\tau$ and $U$ is ancillary. Consider a nonrandomized rule $\delta(T, U)$ based on the sufficient statistic $(\bar{X}, \bar{Y})$ which is equivariant under the transformation

$$
\left(\begin{array}{c}
z \\
\omega
\end{array}\right)=\left(\begin{array}{ll}
c & 0 \\
0 & \frac{1}{c}
\end{array}\right)\left(\begin{array}{l}
\bar{X} \\
\bar{Y}
\end{array}\right) ; c>0
$$

For $\delta(T, U)$ to be scale equivariant, we must have

$$
c \delta(T, U)=\delta(\mathrm{c} T, U) \quad ; \quad \forall c>0
$$

Following Lehman [3] a necessary and sufficient condition for an estimator $\delta$ to be scale equivariant is that it is of the form $\delta=\delta_{0} Z$, where $\delta_{0}$ satisfies (5.2), hence $\delta_{0}=T, Z=\phi(U)$. We see that all the scale equivariant estimators $\delta(T, U)$ must have the form

$$
\delta(T, U)=T \phi(U)
$$

using the loss function (1.2) and the fact that the joint distribution of $\left(\frac{T}{\tau}, \mathrm{U}\right)$ is independent of $\tau$, and we can evaluate the risk at $\tau=1$. Hence

$$
R(\tau, T \phi(U))=E_{U}\left[E\left(1-e^{1+a(T \phi(U)-1)-e^{a(T \phi(U)-1)}}\right) \mid U\right]
$$

It follows that $R(\tau, T(\phi(U))$ is minimized by minimizing the inner expectation. Hence, the minimum risk scale equivariant estimator is $\hat{\tau}_{M R E}=T \phi^{*}(U)$, where $\phi^{*}(U)$ must satisfy the following integral equation 


$$
\int_{0}^{\infty} e^{\left(2 \mathrm{a} \phi^{*}(\mathrm{u})-\mathrm{u}\right) t-\frac{u}{t}-e^{a\left(\mathrm{t} \phi^{*}(u)-1\right)}} \mathrm{d} t=e^{a} \int_{0}^{\infty} e^{\left(\mathrm{a} \phi^{*}(\mathrm{u})-\mathrm{u}\right) t-\frac{u}{t}-e^{a\left(\mathrm{t} \phi^{*}(u)-1\right)}} \mathrm{d} t
$$

where we use the fact that the joint density function of $(\mathrm{T}, \mathrm{U})$ is $\mathrm{g}(\mathrm{t}, \mathrm{u})$, when $t=1$, and [2]

$$
g\left(t, \frac{u}{\tau}\right)=\left\{\begin{array}{cl}
\frac{2 e^{-n u\left(\frac{t}{\tau}+\frac{\tau}{t}\right)} u^{2 n-1}}{n^{-2 n}[(n-1) !]^{2} t} & \text { if } \quad t>0, \mathrm{u}>0 \\
0 & \text { otherwise. }
\end{array}\right.
$$

For deriving the Bayes estimator of $\tau$, let us consider the Inverted Gamma distribution as a prior distribution

$$
\pi_{\alpha, \lambda}(\tau)=\frac{\lambda^{\alpha} e^{-\lambda / \tau}}{\tau^{\alpha+1} \Gamma(\alpha)} ; \tau>0, \lambda>0 .
$$

Therefore the unique Bayes estimator $\delta_{B}$ which is admissible under the loss (1.2) must satisfy the following integral equation

$$
\int_{0}^{\infty} \tau^{-\alpha} e^{\left(2 \mathrm{a} \delta_{B}-\frac{u}{t}\right) \tau-(\lambda+u t) \frac{1}{\tau}-e^{a\left(\tau \delta_{B}-1\right)}} \mathrm{d} \tau=e^{a} \int_{0}^{\infty} \tau^{-\alpha} e^{\left(\mathrm{a} \delta_{B}-\frac{u}{t}\right) \tau-(\lambda+u t) \frac{1}{\tau}-e^{a\left(\tau \delta_{B}-1\right)}} \mathrm{d} \tau
$$

Note that $\hat{\tau}_{M R E}=\hat{\tau}_{B}$, whenever $\alpha \rightarrow 0, \lambda \rightarrow 0$. This means that when the loss function is scale invariant loss $(1.2)$, then $\hat{\tau}_{M R E}$ is a generalized Byes rule against the scale invariant improper prior $\pi(\tau)=\frac{1}{\tau} ; \tau>0$ and is therefore minimax.

\section{References}

1. Fisher, R. A. (1959): Statistical Method and Scientific Inference. London, Oliver and Boyd.

2. Joshi, s. and Nabar, S. (1987): Estimation of the Parameter in the Problem of the Nile. Commun. Statist. Theory Meth. 16, 3149-3156.

3. Lehman, E.L. and Casella, G. (1998): Theory of Point Estimation. Springer-Varlag New York, Inc.

4. Leon, R. V. and Wu, C.F.G. (1992): A Theory of Performance Measure in Parameter Design. Statist. Sinica, 2(2), 335-357.

5. Tribus, M. and Szonya, G. (1989): An Alternate View of the Taguchi Approach. Quality progress, May, 46-52.

6. Varian, H. R. (1975): A Bayesian Approach to Real Assessment. In: Studies in Bayesian Econometric and Statistics in Honor of Leonard J. Savage, eds. S.E. Fienberg and A. Zellner. North Holland, Amesterdam, 195-208.

7. Zellner, A. (1986): Bayesian Estimation and Prediction Using Asymmetric Loss Function. J. Amer Statist. Assoc., 81,446-451. 